\title{
Reconfigurable ultra-wideband transmitter for generation of arbitrary impulse shapes and modulation schemes
}

\author{
A. T. Ott ${ }^{1}$, C. J. Eisner ${ }^{1}$, M. D. Blech ${ }^{2, *}$, and T. F. Eibert ${ }^{1}$ \\ ${ }^{1}$ Lehrstuhl für Hochfrequenztechnik Technische Universität München 80290 Munich, Germany \\ ${ }^{2}$ Institut für Hochfrequenztechnik Universität Stuttgart, 70550 Stuttgart, Germany \\ *now at: Sensing Systems Laboratory, Sony Deutschland GmbH, 70327 Stuttgart, Germany
}

Correspondence to: A. T. Ott (hft@ei.tum.de)

\begin{abstract}
In this paper a reconfigurable ultra-wideband (UWB) impulse radio (IR) transmitter is presented. The IR signal is synthesized at an intermediate frequency (IF) by employing a multi-Nyquist digital-to-analog converter (DAC) with 12 bit resolution and an update rate of $2.3 \mathrm{GHz}$. Digital generation of signals in a field programmable gate array (FPGA) guarantees very high flexibility of the reconfigurable design. For upconversion to the radio frequency (RF) band, a first order bandpass (BP) sampling concept and an alternative conventional concept with mixer stages, have been realized. The system enables to generate signals with arbitrary modulation schemes and techniques at an external host personal computer (PC) employing MATLAB. Different measurements using a digitizing oscilloscope have been conducted to demonstrate the performance of the transmitter.
\end{abstract}

\section{Introduction}

Since the approval of the UWB frequency band by the Federal Communications Commission (FCC) (FCC, 2002), UWB technology has gained enormous research interest (Reed, 2005). Today UWB multiband orthogonal frequency division multiplexing (OFDM) (Batra et al., 2004) and direct sequence spread spectrum (DSSS) systems (Fisher et al., 2004) have established itself in the market. Actually only a few companies like Time Domain (Time Domain Corporation, 2011) or Decawave (DecaWave, 2011) provide IR systems commercially. Their products are characterized by a very low power consumption and they offer the possibility of high resolution tracking combined with low data rate communication.

For planning and evaluation purposes, reconfigurable transceiver designs are an ideal choice as they offer a considerable flexibility with respect to different applications. Recently, software defined radio (SDR) concepts providing common IR modulation schemes and adjustable data rates have been published (Verhelst et al., 2008). For the trans- mission of IR signals in the RF band, direct conversion UWB concepts have been presented, which overcome the problems of non ideal mixer stages (Anderson, 2006; Chen and Brodersen, 2007; Keller et al., 2007). However these concepts are mainly focusing on the receiver implementation whereas adjustable analog pulse generators are used on the transmitter side.

The design and realization of an exemplary subsampling UWB direct conversion SDR IR transceiver has been presented in (Blech et al., 2009) and (Blech et al., 2010). This concept includes a SDR receiver and transmitter solution which increases the flexibility and reduces the impact of non ideal hardware significantly. However the focus of research was on the design of the hardware platform and only binary phase shift keying (BPSK) and quadrature phase shift keying (QPSK) modulation schemes have been analyzed by measurements.

An improved redesign of the transmitter with considerably increased capabilities of the digital signal processing (DSP) stage is presented in this paper. The novel design offers the possibility to generate arbitrary 2-ary and 4-ary IR modulation schemes on an external host PC with MATLAB as programming language. Main parts of the transmitter are an FPGA (Avnet, 2006) and an ultra high speed multi-Nyquist DAC (Maxim Integrated Products, Inc., 2006). Hence direct conversion of the output signal to RF is possible by applying a bandpass filter. Furthermore, an additional frontend with analog mixer stages can be used for upconversion to RF of the analog UWB signal, synthesized at an intermediate frequency (IF). Due to hardware limitations the prototype is designed for laboratory use. The prototype is currently designed to transmit impulses at a centre frequency of $2.5 \mathrm{GHz}$ and an impulse bandwidth of up to $1 \mathrm{GHz}$. However, the results are used to draw conclusions for an implementation with RF output frequencies between $6 \mathrm{GHz}$ and $10 \mathrm{GHz}$.

This paper presents the hardware design of the transmitter in Sect. 2. In Sect. 3, a signal model for characterizing 
possible IR modulation schemes supported by the transmitter is provided. Measurement results demonstrating the performance of the concept are depicted in Sect. 4 before the paper is concluded in Sect. 5.

\section{Transmitter design}

The DAC features 12 bit resolution at an update rate up to $r_{\mathrm{U}}=2.3 \mathrm{GS} \mathrm{s}^{-1}$ and thus generation of signals of up to $1.15 \mathrm{GHz}$ bandwidth. For upconversion the transmitter either uses first order sampling plus filtering in the 2nd or 3rd Nyquist zone or a conventional architecture with mixer stages. To achieve comparable results, signals are synthesized for the RF frequency range between $2 \mathrm{GHz}$ and $3 \mathrm{GHz}$. This is due to the hardware restrictions of the DAC, as signal distortion increases significantly for the $4^{\text {th }}$ and higher Nyquist zones. The current implementation provides variable pulse repetition frequencies (PRF) reaching from $0.5 \mathrm{MHz}$ to $62.5 \mathrm{MHz}$. In principle higher repetition rates are possible on the transmitter side, however a maximum PRF of $62.5 \mathrm{MHz}$ is reasonable, as the PRF is limited by the correlation receiver due to multipath propagation, which causes overlapping impulses (Win and Scholtz, 1998), (Reed, 2005). Although the transmitter operates outside the approved UWB frequency band, the concept is adequate for testing the performance in a laboratory environment.

\subsection{Digital signal processing architecture}

The block diagram of the DSP stage of the transmitter is depicted in Fig. 1. The clock signal of the DAC is generated externally and has been chosen to $2 \mathrm{GHz}$. A Virtex 5 FPGA has been applied for digital generation of pulse trains. Up to four different impulse shapes are stored in a random access memory (RAM) offering the possibility of arbitrary modulation of the incoming bit stream. To relax timing constraints, the FPGA clock rate for transmitting the digitized waveforms from the FPGA to the DAC has been chosen to $f_{\mathrm{FPGA}}=f_{\mathrm{s}} / 8$. Configuration of the FPGA as well as streaming of data is implemented by connecting an external host PC with a high speed universal serial bus interface (USB) 2.0 controller to the FPGA. By accessing the USB interface of the PC in MATLAB, impulse shapes and modulation schemes can be designed and visualized in MATLAB. The prototype of the developed transmitter is depicted in Fig. 2.

\subsection{Frontend concept}

Block diagrams for signal generation with a conventional and a subsampling concept are illustrated in Fig. 3. The subsampling concept uses replicas of the signal in higher Nyquist zones (see Fig. 3 top) whereas in the conventional concept, mixer stages are applied for shifting signals to the RF band. Both concepts have been realized and investigated in this work.
Each discrete signal theoretically provides a periodic spectrum. However the shape of the replicas in higher Nyquist zones are strongly affected and attenuated by the non-ideal sampling impulse shape of the DAC. For optimization of the output power and gain flatness in different Nyquist zones the DAC exhibits different modes of operation. According to the shape of the impulse response in the time domain the DAC has a so called non-return-to-zero (NRZ), a return-tozero (RZ) and a RF mode (Blech et al., 2009). With respect to the required output frequency the NRZ mode provides the highest power in the first Nyquist zone, the RZ mode offers the flattest gain in the third Nyquist zone whereas the RF mode has the highest output power in the third Nyquist zone. Adjustable power amplifiers (PAs) increase the output signal power according to the effective isotropic radiated power (EIRP) requirements of the regulation.

In general the RF mode is applied in the subsampling concept due to the highest signal-to-noise-ratio (SNR) in the third Nyquist zone. An anti-aliasing bandpass (BP) filter yields the desired output signal in the RF band. As subsampling concepts for UWB transmitters are currently restricted by hardware limitations to signals below $3 \mathrm{GHz}$, mixer stages enable the desired upconversion to frequencies beyond $6 \mathrm{GHz}$. For generation of signals on the IF the NRZ mode is applied. To compare the system performance with the subsampling concept and to keep the oscillator circuit as simple as possible the mixer frontend is designed for RF frequencies between $2 \mathrm{GHz}-3 \mathrm{GHz}$ likewise. Equivalent to subsampling concepts a pseudo-coherent design is achieved by coupling of the oscillator signals of the DAC and the mixer. For upconversion of signals a passive diode ring double-balanced mixer is utilized (Mini-Circuits, 2011). By using a low pass (LP) antialiasing filter and the NRZ mode of the DAC, an IF signal with high SNR and significant suppression of spectral components in higher Nyquist zones is achieved. Using a BP filter for image rejection yields the desired RF output signal.

\section{Analytical rationale of impulse generation}

Finding and testing of appropriate impulse shapes for IR systems under real conditions is one of the purposes of the transmitter design. Fulfilling the EIRP constraints of the FCC modulated Gaussian pulses, Gaussian monocycle pulses or numerically generated pulses (Parr et al., 2003) are practicable for sending data from the transmitter to the receiver. Therefore, a signal model adapted to the hardware capability in terms of telecommunication engineering and signal bandwidth is presented.

Many signal models have been published in recent years (Win and Scholtz, 1998; Romme and Piazzo, 2002; Benedetto and Giancola, 2004). They offer the possibility of predicting the EIRP or to decide whether modulation schemes appear to be feasible for certain applications. With respect to the performance of the presented 


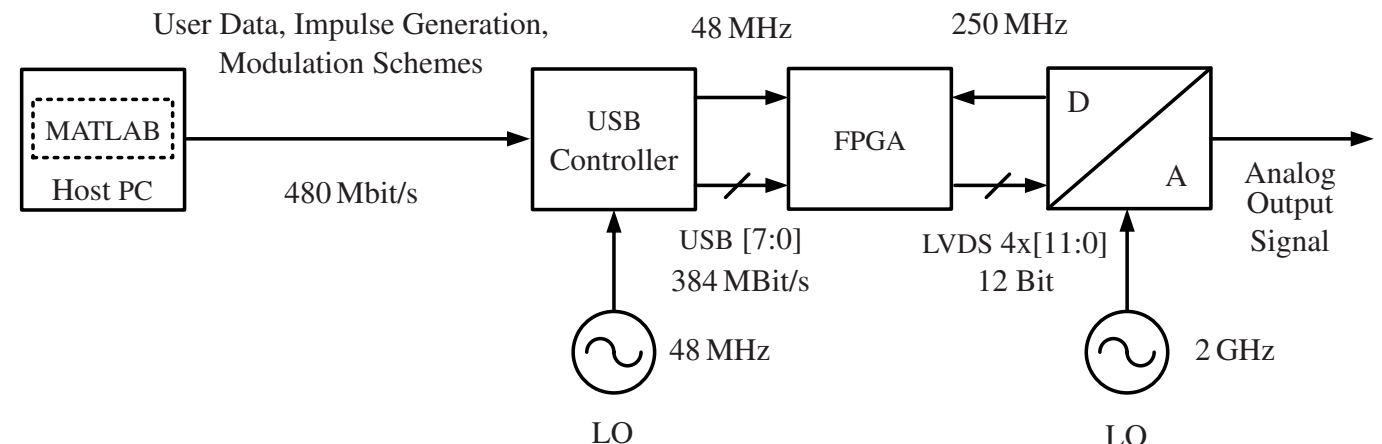

Fig. 1. Block diagram of the digital signal synthesis in the transmitter.

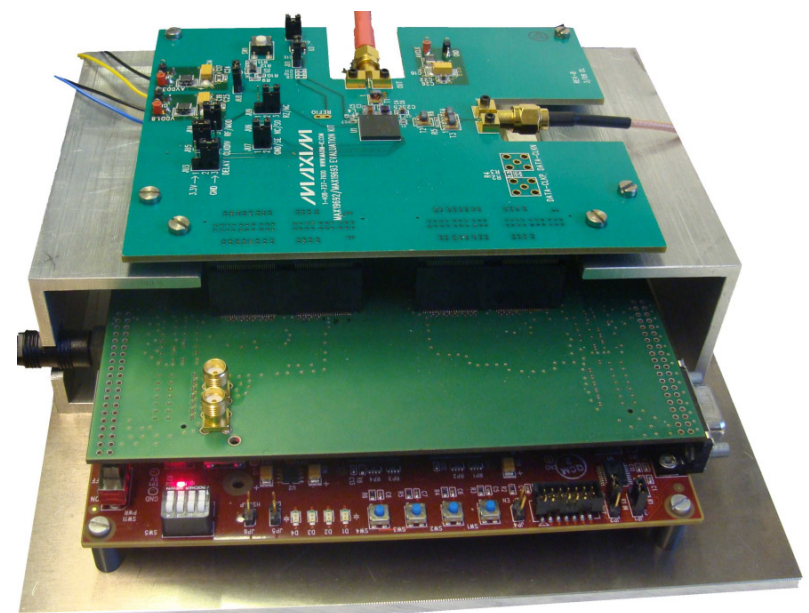

Fig. 2. Developed IR SDR UWB transmitter including FPGA and DAC development boards.

transmitter a signal model similar to (Win and Scholtz, 1998) is introduced. The transmitter supports classical 2-ary and 4-ary modulation schemes like on-offkeying (OOK), pulse-code-modulation (PCM), binary-pulseamplitude-modulation (BPAM), quadrature-pulse-amplitudemodulation (QPAM), pulse-position-modulation (PPM) a combination of 2-ary modulation schemes or novel modulation schemes. Based on 4-ary modulation schemes a mapping of the binary input sequence to the output sequence $c(n) \in\{1,2,3,4\}$ is necessary. Hence the UWB signal

$s(t)=\sum_{n=-\infty}^{\infty} a^{(c(n))} p^{(c(n))}\left(t-n T_{\mathrm{S}}+\epsilon^{(c(n))}\right)$

can be derived from an infinite sum of the amplitude $a^{(c(n))}$, an additional time shift $\epsilon^{(c(n))}$ and pulse shape $p^{(c(n))}(t)$ of each symbol. The pulse repetition time is denoted with $T_{\mathrm{S}}$. Hence the impulse shape of a certain symbol $c(n)$ is
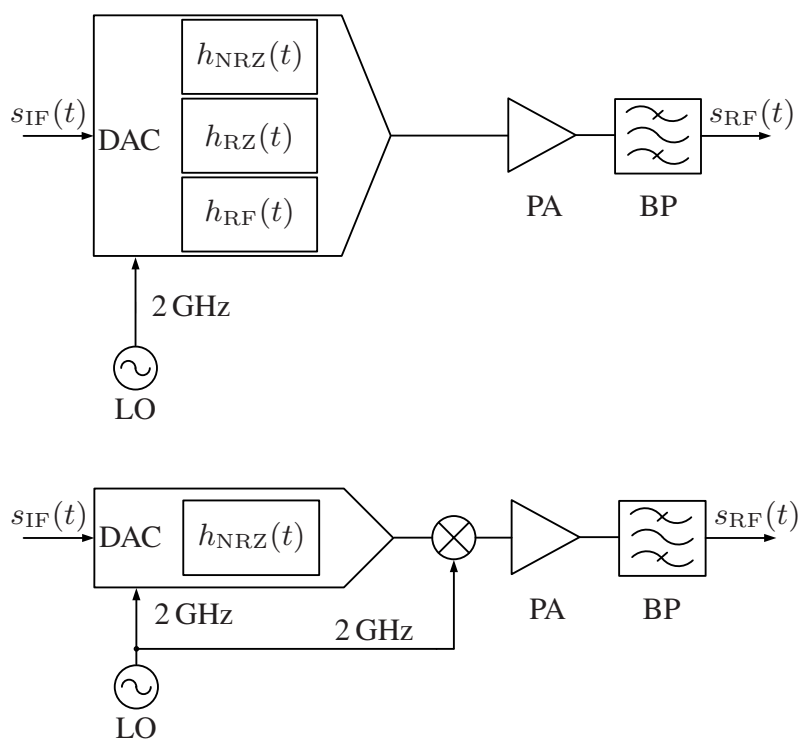

Fig. 3. Block diagram of the digital signal synthesis in the transmitter.

described by

$p_{\mathrm{S}}^{(c(n))}(t)=a^{(c(n))} p^{(c(n))}\left(t+\epsilon^{(c(n))}\right)$

and the resulting transmitted signal

$s(t)=\sum_{n=-\infty}^{\infty} p_{\mathrm{S}}^{(c(n))}\left(t-n T_{\mathrm{S}}\right)$

is obtained from an infinite series of different impulses.

As Gaussian impulses exhibit the smallest time to bandwidth product, they are perfectly suited for UWB applications. In (Blech et al., 2008) a method of defining Gaussian impulses according to the regulatory aspects of the FCC has been published. Following this proposal the shape of Gaussian impulses are defined in the frequency domain and 
adapted to the present signal model. Impulses with a Gaussian shape exhibit Gaussian characteristic in the time and frequency domain. By applying the Fourier transform, Gaussian impulses in the time and frequency domain

$\mathrm{e}^{-\alpha t^{2}} \circ \cdot \sqrt{\frac{\pi}{\alpha}} \mathrm{e}^{-\frac{1}{4 \alpha} \omega^{2}}$

are directly related to each other (Poularikas, 2000). In (4) the factor $\alpha$ scales the shape of the Gaussian impulse with respect to time. To fulfill the regulatory aspects it is evident to determine the amplitude of the time domain output signal. According to the linearity of the Fourier transform the correspondence

$\hat{U} \mathrm{e}^{-\alpha t^{2}} \circ \cdot \hat{U} \sqrt{\frac{\pi}{\alpha}} \mathrm{e}^{-\frac{\pi^{2} f^{2}}{\alpha}}$

is introduced, where $\hat{U}$ denotes the peak amplitude of the time domain signal. According to the FCC regulations UWB impulses have to exceed a bandwidth $B$ of $500 \mathrm{MHz}$ whereby the factor $G_{\mathrm{F}}$ at the cutoff frequency $f_{\mathrm{C}}$ scales the amplitude to $-10 \mathrm{~dB}$ below the maximum amplitude of the impulse. Employing these initial conditions, the unknown variable $\alpha$ is derived from Eq. (5) according to

$\alpha=-\frac{B^{2} \pi^{2}}{4 \ln \left(G_{\mathrm{F}}\right)}$.

Replacing $\alpha$ in Eq. (5) the Gaussian impulse in the frequency domain

$P_{\mathrm{S}}(f)=\hat{U} \sqrt{\frac{-\ln \left(G_{\mathrm{F}}\right)}{\pi(B / 2)^{2}}} \mathrm{e}^{\ln \left(G_{\mathrm{F}}\right)\left(\frac{f^{2}}{(B / 2)^{2}}\right)}$

can be directly adapted to the bandwidth definition of the FCC. In Fig. 4 a Gaussian impulse in the frequency domain with a $-10 \mathrm{~dB}$ bandwidth of $B=500 \mathrm{MHz}$ is depicted. Thus the Gaussian baseband impulse in the time domain

$p_{\mathrm{S}, \mathrm{BB}}(t)=\hat{U} \mathrm{e}^{\frac{(B / 2)^{2} \pi^{2}}{\ln \left(G_{\mathrm{F}}\right)} t^{2}}$

is likewise related to $\hat{U}$ and $B$. Based on Eq. (8) a Gaussian baseband impulse in the time domain is depicted in Fig. 5. As the Gaussian impulses have to be shifted from the baseband to the RF band, the baseband impulse is modulated with the carrier frequency $f_{\mathrm{RF}}$ according to

$p_{\mathrm{S}, \mathrm{RF}}(t)=\hat{U} \mathrm{e}^{\frac{(B / 2)^{2} \pi^{2}}{\ln \left(G_{\mathrm{F}}\right)} t^{2}} \cos \left(2 \pi f_{\mathrm{RF}} t\right)$.

Gaussian waveforms with IF and RF carrier frequencies are also illustrated in Fig. 5. The IF impulse corresponds to the digitally synthesized impulse shape in the first Nyquist zone. The Gaussian impulses at the center frequency $f_{\mathrm{RF}}=2.5 \mathrm{GHz}$ is equivalent to the transmitted output signal.

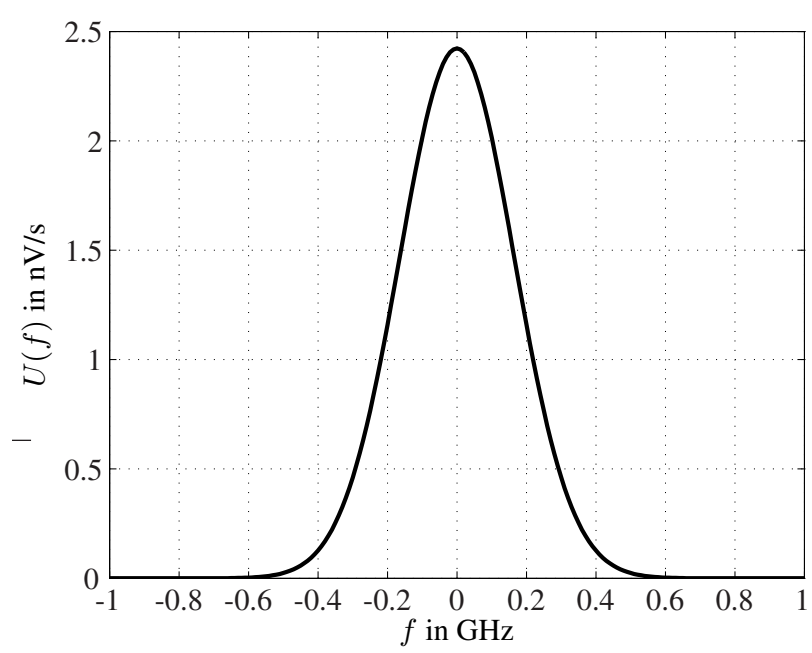

Fig. 4. Gaussian baseband impulse with a $-10 \mathrm{~dB}$ bandwidth of $500 \mathrm{MHz}$ in the frequency domain.

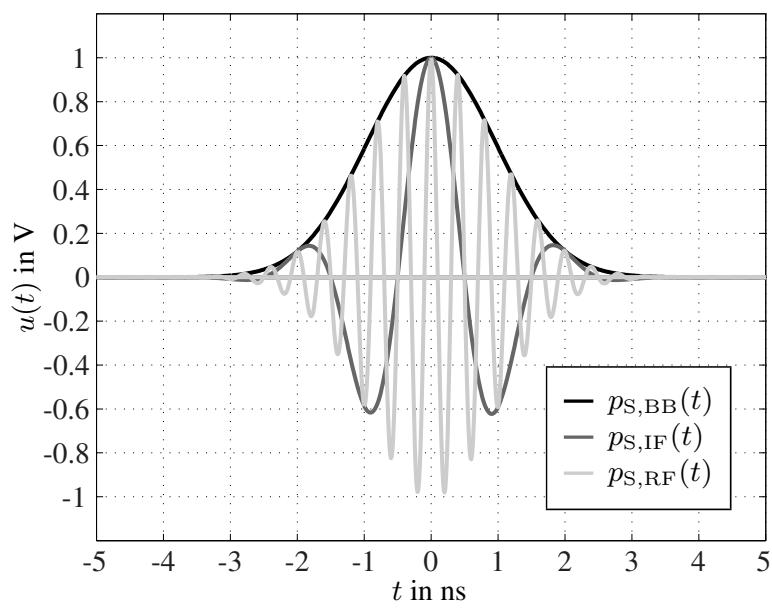

Fig. 5. Gaussian impulse modulated with different carrier frequencies and a $-10 \mathrm{~dB}$ bandwidth of $500 \mathrm{MHz}$ in the time domain.

Comparing the shapes of the baseband and RF Gaussian impulses, it is obvious that the envelope of the RF output signal maintains the shape of the baseband impulse response. The peak amplitude depends on the equivalent isotropically radiated power (EIRP) and is chosen individually for different modulation and coding schemes (Ott et al., 2012).

\section{Results}

Measurements of exemplary modulation schemes have been carried out to show the multifunctional performance of the transmitter. To achieve an obvious distinction between the performance of different modulation schemes, impulses are depicted in the first Nyquist zone and the symbol rate is 


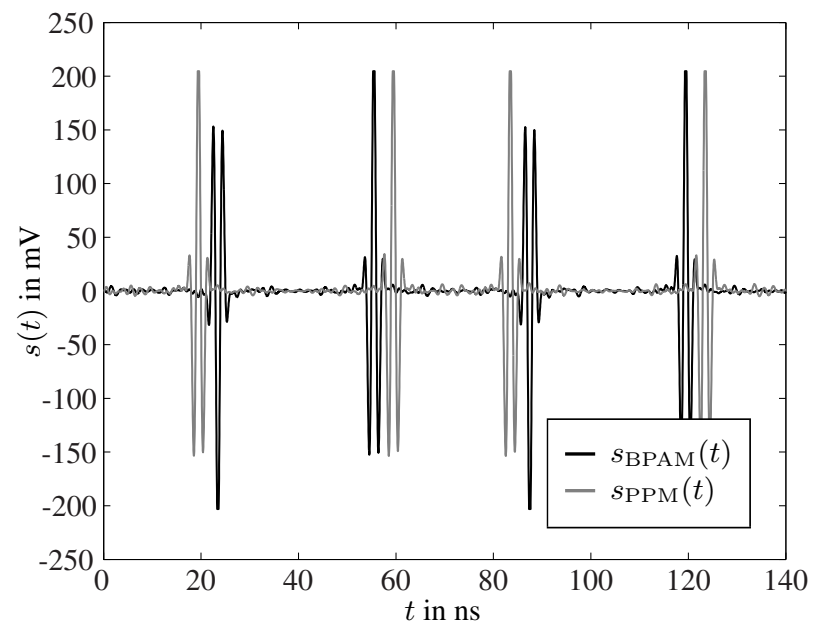

Fig. 6. Measurement of exemplary pulse trains with QPSK modulation scheme and TR-BPAM modulation technique provided by the transmitter.

chosen to $62.5 \mathrm{Mbits}^{-1}$. A LeCroy WaveMaster $808 \mathrm{Zi}-\mathrm{A}$ oscilloscope has been employed for recording pulse trains with different modulation schemes. Representing 2-ary modulation schemes BPAM and PPM have been used and are depicted in Fig. 6. Four impulses of a binary sequence with changing sign have been extracted. As depicted in Fig. 6 sign or position of pulses changes when employing BPAM or PPM, respectively.

In equivalent way 4-ary modulation schemes are applicable by the transmitter. In Fig. 7 four individual waveforms are mapped on the input sequence and hence two bits are transmitted via one impulse. The orthogonal shape of the pulses is achieved by shifting the phase information of the carrier frequency by $90^{\circ}$. This IR modulation scheme has been introduced in (Blech et al., 2008) and can be seen as quadrature-phase-shift-keying (QPSK) pulse modulation or as PCM as every of the four impulses has an individual shape. Furthermore the transmitter enables to produce a combination of two 2-ary modulation schemes. Besides a various number of supported modulation schemes the transmitter offers transmitted reference (TR) modulation technique. Hence the afore mentioned modulation schemes can be additionally adapted to the TR modulation technique e.g. TR-PCM, TRBPAM or TR-PPM. As an example TR-BPAM (TR-BPSK) is depicted in Fig. 7. In a TR system a reference as well as a data impulse is transmitted, whereas the first impulse is employed to demodulate the later impulse by cross correlation. As an advantage, no channel estimation is necessary at the receiver. However, the data rate reduces by a factor of two.

The performance analysis of the frontend is important for the future design of the transmitter. Gaussian impulses synthesized by the subsampling and the conventional concept are presented in Fig. 8. Using the RZ-mode of the DAC re-

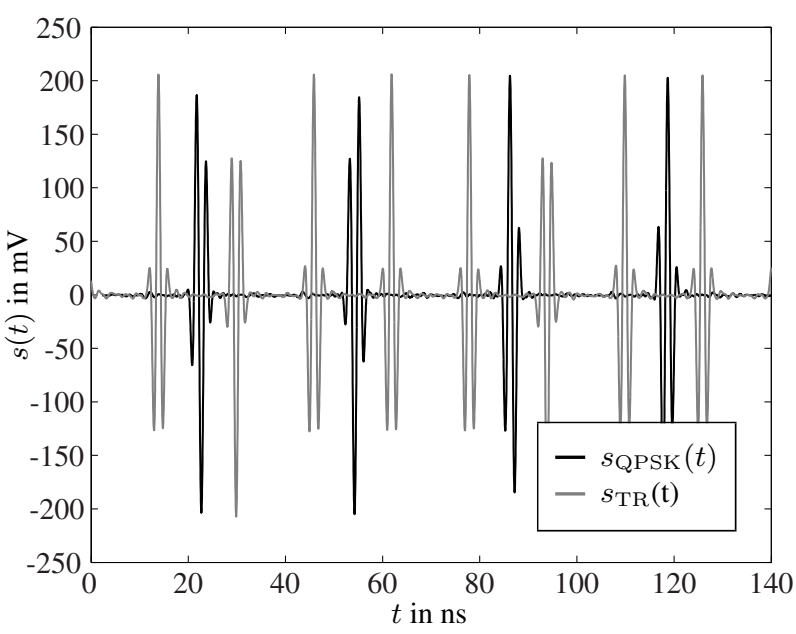

Fig. 7. Measurement of exemplary pulse trains with QPSK modulation scheme and TR-BPAM modulation technique provided by the transmitter.

sults in the best SNR performance of the IR SDR transmitter. Although the frequency response of the DAC is non-ideal, significant distortions of the impulse shape can not be recognized. However, a high fidelity of the Gaussian impulse is achieved by employing a mixer design likewise. As the NRZ mode has been applied for synthesizing the IF signal, a higher output signal amplitude compared to the subsampling concept is achieved. The correlation coefficient

$\rho=\frac{\int_{-\infty}^{\infty} p_{\mathrm{S}, \mathrm{RF}}(t) p_{\mathrm{S}, \mathrm{RF}, \mathrm{M}}(t) \mathrm{d} t}{\sqrt{\int_{-\infty}^{\infty}\left|p_{\mathrm{S}, \mathrm{RF}}(t)\right|^{2} \mathrm{~d} t \int_{-\infty}^{\infty}\left|p_{\mathrm{S}, \mathrm{RF}, \mathrm{M}}(t)\right|^{2} \mathrm{~d} t}}$

of the measured and the ideal Gaussian impulses is a criterion for the performance of the transmitter. In (10) $p_{\mathrm{S}, \mathrm{RF}, \mathrm{M}}(t)$ is either the measured impulse shape of the conventional or the subsampling concept. A very accurate impulse shape of the generated pulses with both frontend concepts is achieved as $\rho_{\mathrm{SDR}}=0.991$ and $\rho_{\text {Mixer }}=0.986$ are the resulting correlation coefficients of the SDR and the conventional concept, respectively.

In order to utilize a transmitter operating in a frequency range between $6 \mathrm{GHz}$ and $10 \mathrm{GHz}$, a pseudo coherent design including mixer stages will be applied in the future. The main reason is that there is currently no DAC available for shifting signals into the UWB frequency band. However, a frontend with high complexity is necessary to guarantee the coherent design, to minimize clock jitter and to avoid signal distortion.

\section{Conclusions}

For many applications IR technology is an appropriate solution. A reconfigurable development platform offers the opportunity for enhanced investigation of IR technology. In this 


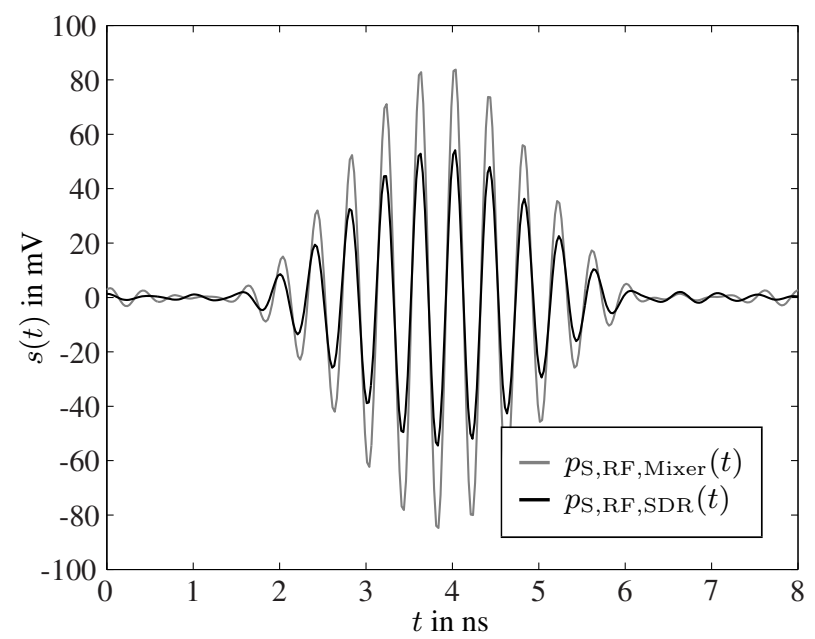

Fig. 8. Typical impulse synthesized by the SDR and the conventional concept in the 3rd Nyquist zone.

paper a prototype of an IR UWB transmitter has been presented. For RF transmissions either a conventional approach including mixer stages or a subsampling approach has been implemented. Both approaches have been compared and it turned out that a solution with a conventional architecture is preferred for the following design of the transmitter. The digital part of the transmitter offers a very high flexibility with respect to telecommunication engineering. Practical measurements of pulse trains with different modulation schemes and techniques have been performed and show the excellent performance of the transmitter.

Acknowledgement. The authors thank LeCroy for supporting the work.

The work described in this paper is related to research activities carried out during the affiliation of $\mathrm{M}$. Blech with the Universität Stuttgart.

\section{References}

Batra, A., Balakrishnan, J., Dabak, A., et al.: IEEE Multi-band OFDM Physical Layer Proposal for IEEE 802.15 Task Group 3a, IEEE P802.15 Wireless Personal Area Networks, 2004.

Anderson, C. R.: A software-defined ultra-wideband transceiver testbed for communications, ranging, and imaging, Ph.D. thesis, Virginia Polytechnic Institute; State University, Blacksburg, VA, USA, 2006.

Avnet: Xilinx Virtex-5 LX50 Development Kit, http://www.avnet. com, 2006.
Benedetto, M. D. and Giancola, G.: Understanding Ultra Wide Band Radio Fundamentals, Prentice Hall, 1 edn., 2004.

Blech, M. D., Geier, D., and Eibert, T. F.: Concept of an UWB impulse radio B-/QPSK transmitter based on standard logic components, in: International Conference on Ultra-Wideband (ICUWB), 1, pp. 225-228, 2008.

Blech, M. D., Neumaier, P., Ott, A. T., Zan, A. A., and Eibert, T. F.: Performance analysis of a software defined subsampling ultra-wideband B-/QPSK impulse radio transceiver, in: European Wireless Technology Conference (EuWIT), 112-115, 2009.

Blech, M. D., Ott, A. T., Neumeier, P., Möller, M., and Eibert, T. F.: A reconfigurable software defined ultra-wideband impulse radio transceiver, Adv. Radio Sci., 8, 67-73, doi:10.5194/ars-8-672010, 2010.

Chen, S. W. and Brodersen, R. W.: A Subsampling Radio Architecture for Ultrawideband Communications, IEEE T. Signal Proces., 55, 5018-5031, 2007.

DecaWave: ScenSor, http://www.decawave.com, 2011.

FCC: Revision of Part 15 of the Commission's Rules Regarding Ultra-Wideband Transmission Systems,http://www.fcc.gov, 2002.

Keller, C. M., Burkhart, J. M., and Phuong, T. T.: Ultra-Wideband Direct Sampling Receiver, in: International Conference on UltraWideband (ICUWB), 387-392, 2007.

Maxim Integrated Products, Inc.: 12-Bit, 2.3Gsps, MultiNyquist DAC with Selectable Frequency Response, http://www. maxim-ic.com, 2006.

Mini-Circuits: ADE-30+ Frequency Mixer, http://minicircuits.com, 2011.

Ott, A. T., Eisner, C. J., and Eibert, T. F.: Enhanced Investigations on Effective Isotropic Radiated Power Emissions of Impulse Radio Devices, in: 6th European Conference on Antennas and Propagation (EuCAP), 1-5, 2012.

Parr, B., Cho, B., Wallace, K., and Ding, Z.: A novel ultra-wideband pulse design algorithm, Communications Letters, 7, 219-221, 2003.

Poularikas, A. D.: The Transforms and Applications Handbook, CRC Press, 2 edn., 2000.

Fisher, R., Kohno, R., Ogawa, H. et al.: DS-UWB Physical Layer Submission to 802.15 Task Group 3a, IEEE P802.15 Wireless Personal Area Networks, 2004.

Reed, J. H.: An Introduction to Ultra Wideband Communication Systems, Prentice Hall, 2005.

Romme, J. and Piazzo, L.: On the power spectral density of timehopping impulse radio, in: Conference on Ultra Wideband Systems and Technologies, 241-244, 2002.

Time Domain Corporation: PulsOn 400 RCM, http://www. timedomain.com, 2011.

Verhelst, M., Ryckaert, J., Vanderperren, Y., and Dehaene, W.: A Low Power, Reconfigurable IR-UWB System, in: International Conference on Communications (ICC), 3770-3774, 2008.

Win, M. Z. and Scholtz, R. A.: Impulse radio: how it works, Communications Letters, 2, 36-38, 1998. 